Hyperactivity Disorder (ADHD) of mixed type through nonlinear EEG synchronization measures.

Methods: Signals: digitised monopolar EEGs (sampling rate: $256 \mathrm{~Hz}$ ) from an ADHA group (12 males, 6-10 years) and a control (CONT) group (11 males, 6-10 years). Recording electrodes: Fp1/C3/ T3/O1 (reference A2) and Fp2/C4/T4/O2 (reference A1). Pairwise interdependence between electrodes was assessed through a nonlinear index $(L)$ for the assessment of generalized synchronization between the amplitudes of the signals. Also, properly generated surrogate data were used to test the significance of the interdependences. Intra cortical connectivity for the left and right hemisphere was obtained averaging all possible intrahemispheric pairs $L$ values. Interhemispheric connectivity was computed averaging the homotopic hemisphere electrodes pairs $L$ values. Conditions considered were: basal eyes-open and basal eyes-closed. ANOVA test for repeated measures and post-hot Bonferroni test was used for between-group comparisons.

Results: Intrahemispheric interdependences did not shown between-groups differences in any experimental conditions considered. Nevertheless, interhemispheric interdependence was greater $(P<$ 0.001) for the ADHA group in both experimental conditions considered without significant differences between them.

Conclusions: ADHD children of mixed type show increased generalized nonlinear interhemispheric synchronization. Nonlinear EEG interhemispheric synchronization measures might be considered as clinical diagnostic help for ADHD children

583

\section{LACTOFERRIN SUPPLEMENTATION PROTECTS BRAIN DEVELOPMENT IN RAT PUPS EXPOSED TO PRENATAL DEXAMETHASONE}

P. Larvaron ${ }^{1}$, Y. Van de Looij ${ }^{1,2}$, E. Somm ${ }^{1}$, B. Wang ${ }^{3}$, R. Gruetter ${ }^{2,4}$, P. Huppi ${ }^{1}$, S. Sizonenko ${ }^{1}$

${ }^{1}$ Department of Pediatrics, University of Geneva, Geneva, ${ }^{2}$ Laboratory for Functional and Metabolic Imaging, Ecole Polytechnique Fédérale de

Lausanne, Lausanne, ${ }^{3}$ Nutrition an Health, Nestlé Research Center, Vers-chez-les-Blancs, ${ }^{4}$ Departments of Radiology, University of Geneva and of Lausanne, Geneva-Lausanne, Switzerland

Background: In rats, in utero exposure to dexamethasone mimics gestational stress and showed reduction in brain weight and myelination. At postnatal day 7 (P7), in vivo 1H-MRS showed alterations of cerebral metabolism in cortex and hippocampus, suggesting impairment of brain structure, energy metabolism and neurotransmitter. Lactoferrin (Lf) an iron-binding glycoprotein secreted in milk with an antioxidant activity is transferred into the the brain after oral administration.

Aims: We hypothesized that Lf could influence brain development and prevent cerebral damages following prenatal exposure to glucocorticoids.

Methods: Rats were treated with DEX $(100 \mu \mathrm{g} / \mathrm{kg} /$ day) during the third week of gestation with access to standard diet (Ctrl-DEX), or Lactoferrin-enriched diet $(1 \mathrm{~g} / \mathrm{kg} /$ day) during gestation and lactation (Lf-DEX). Sham rats not exposed to DEX fed with standard diet (Ctrl-Veh). In vivo 1H-MRSpectroscopy of hippocampus and cortex were acquired at P7 and compared using a Kruskal-Wallis test.

Results: Ctrl-DEX group showed decreased of NAA-NAAG in the hippocampus compared to CtrlVeh group $(2.55 \pm 0.26 \mathrm{mM}$ vs $3.37 \pm 0.10 \mathrm{mM}, p<$ $0.05)$. A decrease of Glu-GIn was observed in both the hippocampus $(3.74 \pm 0.85 \mathrm{mM}$ vs $5.32 \pm 0.66 \mathrm{mM}$, $p<0.05)$ and the cortex $(2.95 \pm 0.66 \mathrm{mM}$ vs $5.62 \pm 0.78 \mathrm{mM}, p<0.05)$. No differences have been found between Lf-DEX group and both Ctrl-Veh and Ctrl-DEX groups.

Conclusions: Variation of NAA-NAAG and Glu-Gln, considered as markers of neuronal integrity and function, confirmed the altered brain development after Dex exposure and revealed the potential protective effect of the lactoferrin in a rat model of prenatal exposure to adverse conditions.

\section{4}

\section{EFFECT OF RESCUE AND PROPHYLAXIS WITH L-ARGININE ON APOPTOTIC CELL DEATH IN NEONATAL RATS}

C. Turkyilmaz ${ }^{1}$, Z. Turkyilmaz ${ }^{2}$, E. Onal ${ }^{1}$, Y. Atalay ${ }^{1}$, F. Soylemezoglu ${ }^{3}$, B. Celasun ${ }^{4}$

${ }^{1}$ Pediatrics, ${ }^{2}$ Pediatric Surgery, Gazi University Medical Faculty, ${ }^{3}$ Pathology, Hacettepe University

Faculty of Medicine, ${ }^{4}$ Pathology, Baskent

University Medical Faculty, Ankara, Turkey

Hypoxic ischemic brain damage presents significant mortality and morbidity rates in newborns. Various animal models of hypoxia-ischemia have made important contribution to the trial of pathogenesis 